\title{
Leptin modulates the effects of acyl CoA:diacylglycerol acyltransferase deficiency on murine fur and sebaceous glands
}

\author{
Hubert C. Chen, ${ }^{1,2,3}$ Steven J. Smith, ${ }^{1,2}$ Bryan Tow, ${ }^{1}$ Peter M. Elias, ${ }^{4}$ \\ and Robert V. Farese, Jr. 1,2,3 \\ ${ }^{1}$ Gladstone Institute of Cardiovascular Disease, San Francisco, California, USA \\ ${ }^{2}$ Cardiovascular Research Institute, \\ ${ }^{3}$ Department of Medicine, and \\ ${ }^{4}$ Department of Dermatology, University of California, San Francisco, California, USA \\ Address correspondence to: Robert V. Farese, Jr., Gladstone Institute of Cardiovascular Disease, \\ San Francisco, California 94141-9100, USA. \\ Phone: (415) 826-7500; Fax: (415) 285-5632; E-mail: bfarese@gladstone.ucsf.edu. \\ Hubert C. Chen and Steven J. Smith contributed equally to this work. \\ Received for publication August 1, 2001, and accepted in revised form November 19, 2001.
}

Acyl CoA:diacylglycerol acyltransferase (DGAT) is a ubiquitously expressed enzyme that catalyzes the final reaction in the major pathways of triglyceride synthesis. Mice lacking DGAT1 $\left(\mathrm{Dgat}^{-/-}\right)$ demonstrate significant changes in lipid metabolism in several tissues, including the skin. Here we report the effects of DGAT1 deficiency on fur and sebaceous glands. Adult $D g a t^{-/}$mice had dry fur and hair loss, which were associated with atrophic sebaceous glands and fur lipid abnormalities. As a result, $D g a t^{-/}$mice had impaired water repulsion and defective thermoregulation after water immersion. These phenotypes were mostly absent in Dgat/- mice with leptin deficiency, indicating an unexpected role for leptin in modulating the skin phenotype. Our findings indicate that DGAT1 plays an important role in normal fur and sebaceous gland physiology and provide evidence that leptin modulates these processes in the skin.

J. Clin. Invest. 109:175-181 (2002). DOI:10.1172/JCI200213880.

\section{Introduction}

Acyl CoA:diacylglycerol acyltransferase (DGAT), a key enzyme in lipid metabolism, catalyzes the final step in the major pathways of triglyceride synthesis (1-3). Two DGAT enzymes (DGAT1 and DGAT2) have been identified $(3,4)$. DGAT1 activity is widely distributed, and its gene is expressed in all tissues examined $(3,5)$. To determine the biological functions of DGAT1, we disrupted its gene (Dgat) in mice (6). Dgat-deficient $($ Dgat $/-)$ mice are viable and healthy, in part because they have alternative mechanisms for triglyceride synthesis (4). In addition, they are resistant to diet-induced obesity through a mechanism involving increased energy expenditure (6).

While studying the metabolic effects of DGAT1 deficiency in mice, we observed abnormalities in their skin. Here we describe functional and morphological abnormalities in the fur and sebaceous glands of Dgat ${ }^{-1}$ mice. In addition, we report an unexpected, related finding: DGAT1 deficiency had virtually no effects on the fur and sebaceous glands of leptin-deficient $(o b / o b)$ mice, revealing an interaction between DGAT1 and leptin in the skin. Our findings reveal a newly identified role for DGAT1 in fur and sebaceous gland physiology and identify the skin as a site for the actions of leptin.

\section{Methods}

Mice. Dgat ${ }^{-1-}$ mice in C57BL/6 background were generated and genotyped as described (6). Wild-type $\left(\right.$ Dgat $\left.t^{++}\right), o b /+$, and Agouti yellow $\left(A^{Y} / a\right)$ mice (all in C57BL/ 6 background) were from The Jackson Laboratory (Bar Harbor, Maine, USA). DGAT1 deficiency was introduced into $o b / o b$ and $A^{Y} / a$ mice through breeding. $\mathrm{Ob} / \mathrm{ob}$ mice lack leptin and, as a result, are obese and diabetic (7). $A^{Y} / a$ mice are obese because of the ectopic production of agouti-signaling protein, which antagonizes the effects of melanocytestimulating hormone in the hypothalamus (8). $A^{Y} / a$ mice have a functional leptin pathway, although they are leptin-resistant (9). Mice were housed in a pathogen-free barrier facility (12-hour light/dark cycle) and fed rodent chow (Ralston Purina Co., St. Louis, Missouri, USA).

Water repulsion and temperature measurements. Mice were immersed in $37^{\circ} \mathrm{C}$ water for 3 minutes and placed on a paper towel for about 5 seconds to absorb excess water. The mice were then exposed to ambient temperature (about $20^{\circ} \mathrm{C}$ ), and their weights and temperatures were recorded for 30-60 minutes. Core body temperature was measured rectally with a digital thermometer (model 4600; Yellow Springs Instruments Co., Yellow Springs, Ohio, USA). 



d



Leptin infusion and testosterone administration. For peripheral (subcutaneous) infusion, a micro-osmotic pump (DURECT Corp., Cupertino, California, USA) was implanted in the interscapular region. The pump delivered recombinant human leptin (a gift from F. Chehab, University of California, San Francisco) at $250 \mathrm{ng} / \mathrm{h}$ for 14 days. This dose restores a normal plasma leptin level in mice with leptin deficiency resulting from lipodystrophy (10). For central (intracerebroventricular) infusion, a cannula (Brain Infusion Kit II, DURECT Corp.) was attached to the implanted micro-osmotic pump, and the needle was inserted $0.5 \mathrm{~mm}$ caudal and $1 \mathrm{~mm}$ lateral to the bregma (11). Leptin $(10 \mathrm{ng} / \mathrm{h})$ was infused for 14 days. This infusion rate does not affect plasma leptin concentrations (9). Testosterone propionate (Sigma Chemical Co., St. Louis, Missouri, USA) was dissolved in vegetable oil and injected subcutaneously.

In situ hybridization. In situ hybridization was performed as described (12). Briefly, skin sections from wild-type mice were deparaffinized and fixed in $4 \%$ paraformaldehyde. After proteinase $\mathrm{K}$ digestion, the sections were hybridized at $55^{\circ} \mathrm{C}$ for 12 hours with ${ }^{35}$ S-labeled antisense or sense DGAT1 RNA probes. The sections were washed for 20 minutes in $2 \times$ SSC, $10 \mathrm{mM} \beta$-mercaptoethanol, and $1 \mathrm{mM}$ EDTA, treated with RNase A ( 20 $\mu \mathrm{g} / \mathrm{ml})$, and washed at high stringency $(0.1 \times \mathrm{SSC}, 10 \mathrm{mM}$ $\beta$-mercaptoethanol, and $1 \mathrm{mM}$ EDTA) for 2 hours at $60^{\circ} \mathrm{C}$. The sections were dehydrated, dipped in photographic emulsion $\mathrm{NTB}_{2}$ (Eastman Kodak Co. Scientific Imaging Systems, Rochester, New York, USA), and stored

\section{Figure 2}

Abnormalities of water repulsion and thermoregulation in DGAT1-deficient $A^{Y} / a$ but not $o b / o b$ mice. (a and $\mathbf{b}$ ) Effect of DGAT1 deficiency on water repulsion (a) and thermoregulation (b) of $A^{Y} / a$ mice after water immersion. (c-h) Effect of DGAT1 deficiency on water repulsion and thermoregulation of $o b / o b$ mice after water immersion. (c and d) No leptin infusion. (e and $\mathbf{f}$ ) After 2 weeks of subcutaneous leptin infusion (+ Peripheral leptin). ( $\mathbf{g}$ and $\mathbf{h}$ ) Two weeks after the leptin infusion was stopped (After leptin). For each experiment, $n=4$ per genotype. ${ }^{*} P<0.05$.

\section{Figure 1}

Effects of DGAT1 deficiency on fur appearance, water repulsion, and thermoregulation in mice. (a) Dry fur and hair loss in a 16-week-old male Dgat ${ }^{-/-}$mouse. (b) Male Dgat ${ }^{+/+}$and Dgat ${ }^{/-}$mice 5 minutes after water immersion. (c and $\mathbf{d}$ ) Impaired water repulsion and thermoregulation in Dgat ${ }^{-/-}$mice after water immersion. Dgat ${ }^{-1-}$ mice retained more water in their fur than did $D g a t^{+/+}$mice, as reflected by a greater increase in mean relative body weight (c). Dgat ${ }^{/-}$mice also developed hypothermia (d). $n=4$ per genotype. ${ }^{*} P<0.05$.

at $4^{\circ} \mathrm{C}$. After 8 weeks of exposure, the sections were developed and counterstained with hematoxylin and eosin.

Histology. Skin samples were fixed overnight in buffered formalin and embedded in paraffin. Sections were stained with hematoxylin and eosin.

Extraction and analysis of fur lipids. Hair lipids were extracted as described (13). Briefly, about $150 \mathrm{mg}$ of fur was clipped from the back of the mouse and treated twice with $20 \mathrm{ml}$ of acetone for 15 minutes. Lipid extracts were filtered, dried under $\mathrm{N}_{2}$, and resuspended in chloroform. Samples $(150 \mu \mathrm{g}$ of the dried lipids) were loaded on a TLC plate (Silica Gel 60; Sigma-Aldrich, St. Louis, Missouri, USA) and resolved with hexane/ethyl ether/acetic acid (80:20:1 $\mathrm{vol} / \mathrm{vol} / \mathrm{vol})$ or hexane/benzene $(55: 45 \mathrm{vol} / \mathrm{vol})$. The latter system allowed a better separation of nonpolar lipids. For transesterification, the lipid was scraped from the TLC plate, incubated with methanolic acid/toluene (4:1 vol/vol) at $37^{\circ} \mathrm{C}$ for 12 hours, and extracted twice with hexane $(1.5 \mathrm{ml})$. For visualization of lipids, the TLC plate was either exposed to iodine vapor or sprayed with cupric sulfate $(3 \%) /$ phosphoric acid (8\%) and charred at $150^{\circ} \mathrm{C}$ for







Figure 3

DGAT1 mRNA expression in skin. An antisense probe detected DGAT1 mRNA expression in sebaceous glands (arrows, left panel) of skin from wild-type mice. Specific hybridization was not detected by the control sense probe (right panel).

color development. For 1,2-diol staining, the plate was sprayed with $1 \%$ lead tetraacetate (dissolved in benzene), followed by $0.05 \%$ pararosaniline (dissolved in acetic acid/acetone, 1:9 vol/vol) (14).

Real-time PCR. Skin was homogenized, and total RNA was extracted (RNA STAT; Tel-Test Inc., Friendswood, Texas, USA). Primer and probe sequences (actin forward primer: $5^{\prime}$-CATCTTGGCCTCACTGTCCA-3', reverse primer: $5^{\prime}$-GGGCCGGACTCATCGTACT- 3 ', probe: $5^{\prime}$-CTTCCAGCAGATGTGGATCAGCAAGC-3'; DGAT2 forward primer: $5^{\prime}$ AGTGGCAATGCTATCATCATCGT-3', reverse primer: $5^{\prime}$ AAGGAATAAGTGGGAACCCAGATCA-3', probe: $5^{\prime}$-CCTGGCAAGAACGCAGTCACCCTG-3') were selected with Primer Express software (Perkin-Elmer Applied Biosystems, Foster City, California, USA). RNA $(1 \mu \mathrm{g})$ was reverse-transcribed in a $20-\mu \mathrm{l}$ reaction containing oligo $(\mathrm{dT})_{12-18}$ primer and Superscript II enzyme (Invitrogen Corp., Carlsbad, California, USA). Each PCR $(50 \mu \mathrm{l})$ contained $1 \mu \mathrm{l}$ of cDNA, $1 \times$ gold buffer II, $4 \mathrm{mM} \mathrm{MgCl} 2,500 \mu \mathrm{M}$ dNTP, primers (200 nM), $100 \mathrm{nM}$ probe (labeled with 6-carboxyfluorescein), and 1.25 U AmpliTaq Gold DNA polymerase (Perkin-Elmer Applied Biosystems). Realtime PCR was performed and analyzed with the ABI Prism 7700 Sequence Detection System (Perkin-Elmer Applied Biosystems). Relative expression levels were calculated by the comparative $\mathrm{C}_{\mathrm{T}}$ (cycle of threshold detection) method as outlined in the manufacturer's technical bulletin; $\beta$-actin expression was used as control.

Statistical analysis. Data are expressed as mean \pm SD. Differences in weight and temperature curves were compared by ANOVA followed by the Tukey-Kramer test, as appropriate.

\section{Results}

Fur abnormalities and impaired water repulsion in Dgat ${ }^{-1}$ mice. Dgat ${ }^{-1}$ mice had normal fur appearance at weaning. After puberty (age 6-8 weeks), however, the fur of $D$ gat ${ }^{-1}$ mice appeared drier and displayed a less prominent sheen than that of $D g a t^{+/+}$mice (Figure 1a). Hair loss also occurred thereafter (Figure 1a), beginning on the dorsal surface of the neck and proceeding caudally. Hair loss was more prominent in male mice than in female mice. Heterozygous $\left(\right.$ Dgat $\left.^{+/}-\right)$mice appeared normal.

Because of their fur abnormalities, we tested the ability of $D \mathrm{gat}^{-/-}$mice to repel water and maintain normal body temperature when wet. Five minutes after water immersion, $D \mathrm{gat}^{-/-}$mice appeared wetter than $D g a t^{+/+}$ mice, which were nearly dry (Figure $1 \mathrm{~b}$ ). The delayed drying in $D g a t^{-/-}$mice resulted from increased water absorption during water immersion (Figure 1c). Dgat ${ }^{-/}$mice became lethargic and exhibited little grooming behavior after water immersion, most likely because of hypothermia, which persisted for more than 60 minutes (Figure 1d). The decreased grooming probably also contributed to their delayed drying. Dry Dgat/- mice had no thermoregulatory defects. They had core body temperatures comparable to those of $\mathrm{ggat}^{+/+}$mice, both at room temperature $\left(20^{\circ} \mathrm{C}\right)$ and during prolonged cold exposure (24 hours at $4^{\circ} \mathrm{C}$, not shown).

Fur abnormalities in DGAT1-deficient $A^{Y} /$ a but not ob/ob mice. In a separate study to examine the metabolic effects of DGAT1 deficiency, we had introduced DGAT1 deficiency into two strains of genetically obese mice, $o b / o b$ and $A^{Y} / a$. DGAT1 deficiency was associated with dry fur and hair loss in $A^{Y} / a$ mice but had little impact on the fur of $o b / o b$ mice. In addition, $A^{Y} / a$ mice with DGAT1 deficiency $\left(D g a t^{-/} A^{Y} / a\right)$ retained more water than did wild-type $\left(D g a t^{+/}\right) A^{Y} / a$ mice (Figure 2a) and developed hypothermia (Figure 2b) after water immersion. In contrast, DGAT1 deficiency in $o b / o b$ mice did not affect water repulsion (Figure $2 c$ ) or thermoregulation (Figure 2d).

To further explore whether the effects of DGAT1 deficiency on fur required leptin, we administered leptin to $o b / o b$ mice with or without DGAT1 $\left(D g a t^{+/+} o b / o b\right.$ or Dgat/-ob/ob). After 2 weeks of continuous peripheral leptin infusion, $D g a t^{-/} o b / o b$ mice exhibited impaired water repulsion (Figure 2e) and developed mild hypothermia (Figure 2f) after water immersion. These findings were again absent 2 weeks after the cessation of leptin infusion (Figure 2, $g$ and $h$ ).



\section{Figure 4}

Age modulates the effect of DGAT1 deficiency on sebaceous gland morphology. (a and $\mathbf{b}$ ) In 6-week-old male mice, the sebaceous glands (SG) and hair follicles (HF) appeared to be normal, regardless of Dgat genotype. (c and d) In 3-month-old male mice, DGAT1 deficiency was associated with atrophic sebaceous glands; for most hair follicles, sebaceous glands were not identifiable. Bar, $30 \mu \mathrm{m}$. 




Figure 5

Sebaceous gland abnormalities in DGAT1-deficient $A^{Y} / a$ but not $o b / o b$ mice. (a and b) Skin section from $D g a t^{+/+} A^{Y} / a$ mice (a) and $D g a t^{1-} A^{Y} / a$ mice (b). (c-j) Skin sections from $D g a t^{+/+} o b / o b$ and Dgat $/-o b / o b$ mice. (c and $\mathbf{d}$ ) No leptin infusion. (e and $\mathbf{f}$ ) After 2 weeks of subcutaneous leptin infusion (+ Peripheral leptin). ( $\mathbf{g}$ and $\mathbf{h}$ ) After 2 weeks of intracerebroventricular leptin infusion (+ Central leptin). (i and $\mathbf{j})$ Two weeks after the leptin infusion was stopped (After leptin). Representative samples from male mice are shown. SG, sebaceous gland. Bar, $30 \mu \mathrm{m}$.

Sebaceous gland atrophy in Dgat/- mice. To investigate the function of DGAT1 in the skin, we examined DGAT1 expression by in situ hybridization, which revealed high DGAT1 mRNA levels in the sebaceous glands (Figure 3). We therefore examined sebaceous gland morphology in Dgat ${ }^{-1-}$ mice. The sebaceous glands and hair follicles of young (6-week-old) $D g a t^{-/}$ mice appeared normal (Figure 4, a and b). In contrast, the skin of older (3-month-old) Dgat ${ }^{-1-}$ mice had atrophic sebaceous glands on both the ventral and the dorsal surfaces (Figure 4, c and d). For many hair follicles, no associated sebaceous glands could be identified.

Atrophic sebaceous glands in DGAT1-deficient $A^{Y} /$ a mice and leptin-treated ob/ob mice. Similar to the situation for fur abnormalities, the sebaceous gland atrophy associated with DGAT1 deficiency was present in $A^{Y} / a$ mice (Figure 5, a and b) but not in $o b / o b$ mice (Figure 5, $\mathrm{c}$ and d). $D g a t^{+/+} o b / o b$ mice, however, had larger sebaceous glands than did $D g a t^{-/}-o b / o b$ mice. Two weeks of periph- eral or central leptin infusion decreased the size of sebaceous glands in $D g a t^{+/+} o b / o b$ mice (Figure 5, e and g) but caused marked atrophy of sebaceous glands in $D g a t^{-/} o b / o b$ mice (Figure 5, f and h). These histological changes reverted to pretreatment states 2 weeks after the cessation of leptin administration (Figure 5 , $\mathrm{i}$ and $\mathrm{j}$ ).

Abnormal fur lipids in Dgat ${ }^{-1}$ mice. We analyzed the effects of DGAT1 deficiency on the composition of fur lipids, which are produced by sebaceous glands (15). In both $D g a t^{+/+}$and $D g a t^{/-}$mice, the fur lipids contained sterol esters, free cholesterol, and triglycerides. In addition, the fur of adult $D g a t^{+/+}$mice contained several lipids that were lacking in the fur of adult $D g a t /-$ mice (Figure 6a). The most prominent of these missing lipids was slightly more polar than sterol esters. After transesterification, this lipid yielded two products - one migrated similarly to a fatty acid methyl ester standard, and the other contained a 1,2-diol group (not shown). Based on this result, as well as the migration and quantity of the lipid on TLC, it is most likely a type II wax diester, the most abundant component in murine fur lipids (16). This difference in fur lipid content was agedependent: it was less striking in younger (6-week-old) mice (Figure 6b, lanes 1 and 2 ) and more pronounced in adult (3-month-old) mice (Figure 6b, lanes 3 and 4).

Abnormal fur lipids in DGAT1-deficient $A^{Y} /$ a mice and leptin-treated $o b / o b$ mice. The fur of $D g a t^{-1}-A^{Y} / a$ mice also contained little, if any, of the wax diester (Figure 6b, lanes 5 and 6). In contrast, both $D g a t^{+/+} o b / o b$ and $D g a t / o b / o b$ mice produced this fur lipid, although the quantity was slightly decreased in the fur of $D g a t /-o b / o b$ mice (Figure 6b, lanes 7 and 8). Two weeks of peripheral or central leptin administration had a minimal effect on the abundance of this fur lipid in $D g a t^{+/} o b / o b$ mice but caused a severe reduction in Dgat/-ob/ob mice (Figure 6b, lanes 9-12). Two weeks after the withdrawal of leptin, the fur of $D g a t^{-/}-o b / o b$ mice contained this lipid again (Figure 6b, lanes 13 and 14).

Effects of androgens on fur lipids in Dgat / mice. Because the effects of DGAT1 deficiency on the skin were most noticeable in postpubertal male mice, we investigated the role of androgens in mediating these effects. Because $o b / o b$ mice have a defective hypothalamic-pituitarygonadal axis, they do not undergo puberty and have decreased serum testosterone levels (17). This lack of normal testosterone production may ameliorate the effects of DGAT1 deficiency in $o b / o b$ mice. To test this hypothesis, we injected both $D g a t^{+/+} o b / o b$ and Dgat $/-o b / o b$ male mice with a replacement dose of testosterone ( $5 \mu \mathrm{g} / \mathrm{g}$ body weight/day) and assessed its effects on fur lipid content. Two weeks of testosterone treatment did not eliminate the presence of wax diesters in the fur of $D g a t^{/-o b} / o b$ mice (Figure 7, lanes 1-4). Testosterone replacement also did not cause atrophy of sebaceous glands in these mice (not shown).

To further explore whether androgens mediated the effects of DGAT1 deficiency in the skin, we castrated postpubertal $D g a t^{-1}$ male mice. Castration did not restore the normal production of fur lipids in Dgat ${ }^{-1-}$ 
a



b


\section{Figure 6}

Abnormal fur lipid content in Dgat ${ }^{-1}$ mice. (a) Absence of specific lipids in the fur of Dgat ${ }^{-1-}$ mice (white and gray arrows). (b) Effects of leptin on fur lipid content. The putative type II wax diester is indicated with a white arrow. Lipids were analyzed by TLC with hexane/ethyl ether/acetic acid (a) and hexane/benzene (b). Experiments were performed 3-4 times. Representative results are shown.

male mice; rather, it completely eliminated the presence of wax diesters in their fur (Figure 7, lanes 5 and 6). Castration also did not reverse the atrophy of sebaceous glands in these mice (not shown).

Upregulation of DGAT2 expression in the skin of DGAT1deficient ob/ob mice. One possible mechanism by which the skin of $D g a t^{-/} o b / o b$ mice was largely protected from the effects of DGAT1 deficiency could be a compensatory increase in the expression of another DGAT enzyme. We therefore measured the mRNA expression of the recently identified DGAT2 (4) to determine whether its expression was increased in the skin of $D g_{a t}-\alpha o b / o b$ mice. In wild-type mice, DGAT2 mRNA was expressed highly in the skin (not shown). DGAT2 expression was not increased in $D g a t /$ mice and in fact was lower than in $D g a t^{+/+}$mice (Figure 8a). However, leptin-deficient $D g a t^{/-}$and $D g a t^{+/+}$mice had similarly increased levels of DGAT2 expression (Figure 8a). Leptin deficiency, therefore, was associated with a greater upregulation of DGAT2 in the skin of Dgat/- mice than in that of $D g a t^{+/+}$mice (Figure $8 \mathrm{~b}$ ).

\section{Discussion}

Here we report the effects of DGAT1 deficiency on skin and fur of mice. Dgat ${ }^{-1-}$ mice developed dry fur and hair loss after puberty. In addition, they exhibited impaired water repulsion and hypothermia after water immer-

\section{Figure 7}

Effects of androgens on fur lipids in Dgat ${ }^{-/-}$mice. The putative type II wax diester is indicated with an open arrow. Lipids were analyzed by TLC with hexane/benzene. For lanes 3 and 4 , testosterone propionate was injected subcutaneously for 2 weeks. For lanes 5 and 6, fur lipids were extracted 2 weeks after castration. Experiments were performed twice. Representative results are shown. sion. Our results suggest that DGAT1 deficiency affected the development of sebaceous glands, resulting in defective production of fur lipids, in particular type II wax diesters. This defect subsequently caused the morphological and functional changes observed in the fur of $D$ gat $/$ - mice. The hair loss, water repulsion, and sebaceous gland abnormalities were nearly absent in $\mathrm{Dgat}^{-/-}$ mice with leptin deficiency, indicating a newly identified role for leptin in hair and sebaceous gland biology.

Sebaceous glands are lipid-producing structures associated with hair follicles. These holocrine glands secrete lipids (sebum) that coat the hair, and in furry mammals these lipids have been hypothesized to play an important role in water repulsion and thermoregulation (15). Sebaceous lipids include wax esters, wax diesters, sterol esters,

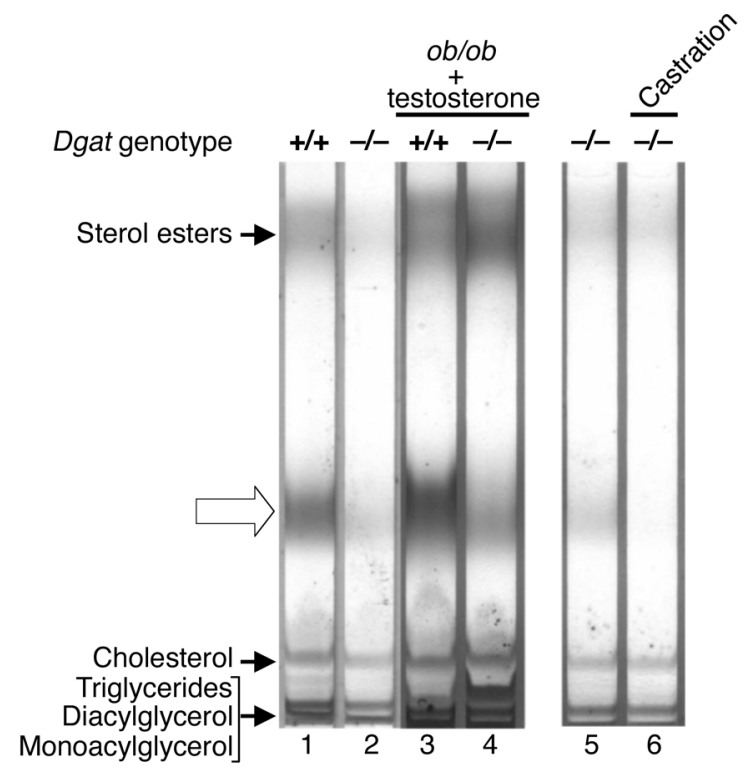






Figure 8

Upregulation of DGAT2 in the skin of $o b / o b$ mice. (a) mRNA expression of DGAT2. $n=3$ per genotype. ${ }^{*} P<0.05$ vs. Dgat ${ }^{+/+}$. (b) Increased upregulation of DGAT2 mRNA expression in $o b / o b$ mice with DGAT1 deficiency. $n=3$ per genotype. ${ }^{*} P<0.05$.

squalene, and triglycerides, but the proportions of these lipids vary significantly among different species (18). For example, triglycerides are a major component of human sebum. In contrast, mouse sebum contains relatively small amounts of triglycerides but is rich in type II wax diesters, which are thought to be important for water repulsion $(15,18)$. The importance of sebaceous glands and sebum is demonstrated by melanocortin-5 receptor-deficient mice, which have defective sebaceous glands that do not produce normal sebum. Consequently, they retain more water in their fur after water immersion and cannot maintain normal body temperature when wet (13).

Our observations suggest that the fur of $D g a t^{/-}$mice lacked type II wax diesters, a major component of murine sebum, most likely because of sebaceous gland atrophy. An alternative explanation is that DGAT1 catalyzed the synthesis of wax diesters and that DGAT1 deficiency resulted in their absence. However, because the fur of $D g a t^{-/}$mice lacked several additional minor lipids, it seems unlikely that all of these were products of the DGAT1 reaction. How DGAT1 deficiency causes sebaceous gland atrophy is unknown. DGAT1-mediated triglyceride synthesis may be essential for normal sebaceous gland development in mice, even though relatively small amounts of triglycerides are secreted in mouse sebum. Another possibility is that DGAT1 deficiency may alter the concentration of reaction substrates (e.g., diacylglycerols, FFAs), which could then interfere with sebaceous gland development, possibly by modulating signaling pathways.

DGAT1 deficiency had minimal effects on fur and sebaceous glands in $o b / o b$ mice. Additionally, infusing leptin either peripherally or centrally into $o b / o b$ mice with DGAT1 deficiency temporarily resulted in impaired water repulsion, deficiency of the fur lipid, and sebaceous gland atrophy. Leptin-treated $D$ gat $/-o b / o b$ mice, however, were relatively insusceptible to hypothermia after water immersion, most likely because of their thick layer of subcutaneous fat. We also found that $o b / o b$ mice had hypertrophic sebaceous glands. These results point to an unexpected role for leptin in modulating sebaceous gland development and sebum production.
Little is known about the effects of leptin on sebaceous gland biology. Leptin, a peptide hormone secreted by adipocytes, reduces food intake, enhances energy expenditure, and increases insulin sensitivity through its hypothalamic receptors (19). However, functional leptin receptors are also expressed outside the CNS (20, 21), and recent studies suggest that leptin exerts direct effects in peripheral tissues $(22,23)$, including the skin (24). Leptin, therefore, could modulate sebaceous gland biology either directly in the periphery or through pathways involving the hypothalamus. To investigate these possibilities, we infused leptin either peripherally or centrally (the latter performed with a dose of leptin that does not affect peripheral leptin concentrations). Interestingly, peripheral and central administration of leptin had similar effects on sebaceous gland histology and fur lipid production. These results suggest that the effects of leptin on sebaceous glands can be mediated entirely through the hypothalamus. To our knowledge, this is the first demonstration of a connection between the hypothalamic leptin pathway and structures in the skin.


\section{Figure 9}

A model of how DGAT enzymes, leptin, and androgens modulate sebaceous gland biology. Our data suggest the existence of at least two pathways involved in maintaining normal morphology and functioning of sebaceous glands. (a) One of these pathways (presumably the major pathway) requires DGAT1, whereas the other pathway (the alternative pathway) may require DGAT2. Androgens stimulate the activity of both pathways, whereas leptin downregulates the activity of the alternative pathway. (b) In DGAT1 deficiency, only the alternative pathway is functional, resulting in sebaceous gland atrophy and decreased production of wax diesters. (c) With leptin deficien$c y$, the activity of the alternative pathway is upregulated to levels comparable to those of the major pathway, leading to sebaceous gland hypertrophy and increased fur lipid production. (d) This upregulation of the alternative pathway can compensate for the loss of DGAT1, thereby accounting for the relatively normal sebaceous gland and fur lipid findings in Dgat/-ob/ob mice. 
The neural pathways that mediate the central effects of leptin on peripheral tissues are mostly unknown (25), although the sympathetic nervous system may be an important mediator (26). One plausible interpretation of our results is that leptin, by activating an efferent pathway such as the sympathetic nervous system, suppresses the expression of another DGAT enzyme in the skin. The absence of leptin would then permit the upregulation of the second DGAT, thereby causing sebaceous gland hypertrophy in $D g a t^{+/+} o b / o b$ mice and preventing sebaceous gland atrophy in $D g a t^{-}-o b / o b$ mice. Indeed, DGAT2 was upregulated to similar levels in $\mathrm{Dgat}^{+/+}$and $\mathrm{Dgat}^{/-}$ mice with leptin deficiency. It is possible that this upregulation may compensate for the loss of DGAT1. Additional studies will be needed to further elucidate the regulation of DGAT2 expression by leptin.

Because the fur and sebaceous gland abnormalities associated with DGAT1 deficiency were most obvious in postpubertal male mice, androgens may have a role in mediating the effects of DGAT1 deficiency on the skin. For example, one possible explanation for the relatively normal skin findings in $D g a t / / o b / o b$ mice is that they have low levels of testosterone. However, injecting these mice with replacement doses of testosterone did not result in sebaceous gland atrophy or abnormal fur lipid production. These results suggest that the hypogonadal state of $D g a t /-o b / o b$ mice was unlikely to be a protective factor against the effects of DGAT1 deficiency. Creating a hypogonadal state in postpubertal $\mathrm{Dgat}^{-/}$ male mice by castration also did not reverse their sebaceous gland atrophy or abnormal fur lipid production. In fact, castration completely eliminated the presence of type II wax diesters on the fur of Dgat ${ }^{-/-}$male mice. These findings are consistent with previous observations that androgens promote sebaceous gland development and sebum production $(27,28)$. These results also suggest that the effects of DGAT1 deficiency on sebaceous glands are not mediated through androgens and do not require normal androgen production.

In summary, our findings identify new roles for DGAT1 and leptin in skin biology. A potential model of how DGAT1 deficiency and leptin affect sebaceous gland biology is shown in Figure 9. An enhanced understanding of the functions of molecules such as DGAT1 and leptin in sebaceous glands and hair follicles may provide insights into dermatological conditions involving the sebaceous gland and hair, such as acne vulgaris and alopecia.

\section{Acknowledgments}

We thank C. Tam and the Cardiovascular Research Institute Core Laboratory (University of California, San Francisco) for performing the in situ hybridization, D. Newland for assistance with histology, S. Ordway and G. Howard for editorial assistance, B. Taylor for manuscript preparation, R. Pitas and P. Wertz for insights and assistance on lipid analysis, E. Epstein and K. Feingold for helpful discussions, and S. Young, M. Schambelan, and $\mathrm{K}$. Weisgraber for comments on the manuscript. This work was supported by the NIH (R01 DK56084), the CardioFellows Foundation, the Endocrine Fellows Foundation, and the J. David Gladstone Institutes.

1. Bell, R.M., and Coleman, R.A. 1980. Enzymes of glycerolipid synthesis in eukaryotes. Annu. Rev. Biochem. 49:459-487.

2. Lehner, R., and Kuksis, A. 1996. Biosynthesis of triacylglycerols. Prog. Lipid Res. 35:169-201.

3. Cases, S., et al. 1998. Identification of a gene encoding an acyl CoA:diacylglycerol acyltransferase, a key enzyme in triacylglycerol synthesis. Proc. Natl. Acad. Sci. USA. 95:13018-13023.

4. Cases, S., et al. 2001. Cloning of DGAT2, a second mammalian diacylglycerol acyltransferase, and related family members. J. Biol. Chem. 276:38870-38876.

5. Farese, R.V., Jr., Cases, S., and Smith, S.J. 2000. Triglyceride synthesis: insights from the cloning of diacylglycerol acyltransferase. Curr. Opin. Lipidol. 11:229-234.

6. Smith, S.J., et al. 2000. Obesity resistance and multiple mechanisms of triglyceride synthesis in mice lacking DGAT. Nat. Genet. 25:87-90.

7. Leibel, R.L., Chung, W.K., and Chua, S.C., Jr. 1997. The molecular genetics of rodent single gene obesities. J. Biol. Chem. 272:31937-31940.

8. Fan, W., Boston, B.A., Kesterson, R.A., Hruby, V.J., and Cone, R.D. 1997. Role of melanocortinergic neurons in feeding and the agouti obesity syndrome. Nature. 385:165-168.

9. Halaas, J.L., et al. 1997. Physiological response to long-term peripheral and central leptin infusion in lean and obese mice. Proc. Natl. Acad. Sci. USA. 94:8878-8883.

10. Shimomura, I., Hammer, R.E., Ikemoto, S., Brown, M.S., and Goldstein, J.L. 1999. Leptin reverses insulin resistance and diabetes mellitus in mice with congenital lipodystrophy. Nature. 401:73-76.

11. El-Haschimi, K., Pierroz, D.D., Hileman, S.M., Bjørbæk, C., and Flier, J.S. 2000. Two defects contribute to hypothalamic leptin resistance in mice with diet-induced obesity. J. Clin. Invest. 105:1827-1832.

12. Meiner, V., et al. 1997. Tissue expression studies of mouse acyl CoA:cholesterol acyltransferase gene (Acact): findings supporting the existence of multiple cholesterol esterification enzymes in mice. J. Lipid Res. 38:1928-1933.

13. Chen, W., et al. 1997. Exocrine gland dysfunction in MC5-R-deficient mice: evidence for coordinated regulation of exocrine gland function by melanocortin peptides. Cell. 91:789-798.

14. Mangold, H.K., Zweig, G., and Sherma, J. 1984. CRC handbook of chromatography. Lipids. Volume 2. CRC Press. Boca Raton, Florida, USA. 309-317.

15. Thody, A.J., and Shuster, S. 1989. Control and function of sebaceous glands. Physiol. Rev. 69:383-416.

16. Stewart, M.E., Downing, D.T., and Strauss, J.S. 1983. Sebum secretion and sebaceous lipids. Dermatol. Clin. 1:335-344.

17. Swerdloff, R.S., Batt, R.A., and Bray, G.A. 1976. Reproductive hormonal function in the genetically obese (ob/ob) mouse. Endocrinology. 98:1359-1364.

18. Nikkari, T. 1974. Comparative chemistry of sebum. J. Invest. Dermatol. 62:257-267.

19. Schwartz, M.W., Woods, S.C., Porte, D., Jr., Seeley, R.J., and Baskin, D.G. 2000. Central nervous system control of food intake. Nature. 404:661-671.

20. Tartaglia, L.A. 1997. The leptin receptor. J. Biol. Chem. 272:6093-6096.

21. Ghilardi, N., et al. 1996. Defective STAT signaling by the leptin receptor in diabetic mice. Proc. Natl. Acad. Sci. USA. 93:6231-6235.

22. Shimabukuro, M., et al. 1997. Direct antidiabetic effect of leptin through triglyceride depletion of tissues. Proc. Natl. Acad. Sci. USA. 94:4637-4641.

23. Unger, R.H., Zhou, Y.-T., and Orci, L. 1999. Regulation of fatty acid homeostasis in cells: novel role of leptin. Proc. Natl. Acad. Sci. USA. 96:2327-2332.

24. Frank, S., Stallmeyer, B., Kämpfer, H., Kolb, N., and Pfeilschifter, J. 2000. Leptin enhances wound re-epithelialization and constitutes a direct function of leptin in skin repair. J. Clin. Invest. 106:501-509.

25. Ducy, P., et al. 2000. Leptin inhibits bone formation through a hypothalamic relay: a central control of bone mass. Cell. 100:197-207.

26. Aizawa-Abe, M., et al. 2000. Pathophysiological role of leptin in obesityrelated hypertension. J. Clin. Invest. 105:1243-1252.

27. Ebling, F.J. 1957. The action of testosterone on the sebaceous glands and epidermis in castrated and hypophysectomized male rats. J. Endocrinol. 15:297-306.

28. Akamatsu, H., Zouboulis, C.C., and Orfanos, C.E. 1992. Control of human sebocyte proliferation in vitro by testosterone and 5-alpha-dihydrotestosterone is dependent on the localization of the sebaceous glands. J. Invest. Dermatol. 99:509-511. 